\title{
A general reduction method for one-loop N-point integrals*
}

\author{
G. Heinrich $^{\mathrm{a}}$, T. Binoth ${ }^{\mathrm{b}}$ \\ ${ }^{\text {a}}$ Laboratoire de Physique Théorique LPT \\ Université de Paris XI, Bâtiment 210, 91405 Orsay, France \\ ${ }^{\mathrm{b}}$ Laboratoire d'Annecy-Le-Vieux de Physique Théorique LAPTH \\ Chemin de Bellevue, B.P. 110, 74941 Annecy-le-Vieux, France
}

\begin{abstract}
In order to calculate cross sections with a large number of particles/jets in the final state at next-to-leading order, one has to reduce the occurring scalar and tensor one-loop integrals to a small set of known integrals. In massless theories, this reduction procedure is complicated by the presence of infrared divergences. Working in $n=4-2 \epsilon$ dimensions, it will be outlined how to achieve such a reduction for diagrams with an arbitrary number of external legs. As a result, any integral with more than four propagators and generic 4-dimensional external momenta can be reduced to box integrals.
\end{abstract}

\section{Motivation}

The study of processes with multi-particle/jet final states is of major importance not only to test perturbative QCD, but also to obtain a precise estimate of the background for search experiments. At the Tevatron, multi-jet cross sections with up to six jets have been measured and compared to tree level QCD predictions [1]. However, leading order predictions are very unstable with respect to variations of the renormalization and factorization scales, such that the NLO corrections are necessary to obtain reliable predictions.

The calculation of e.g. a $2 \rightarrow N-2$ parton process at NLO requires the knowledge of $N$-point scalar and tensor one-loop integrals with massless propagators and external legs, for $e^{+} e^{-} \rightarrow N$ jets one needs $(N+1)$-point integrals with one offshell external leg. These integrals have to be further processed by reducing the tensor integrals to scalar integrals and, for $N \geq 5$, reducing the scalar $N$-point integrals to known integrals with less propagators.

Of course, reduction methods have been worked out and successfully applied before [ [3]-[ 7, but mostly for integrals with massive propagators, as needed e.g. for electroweak radiative

\footnotetext{
*Talk presented at Loops and Legs in Quantum Field The-
} ory, April 2000, Bastei, Germany corrections. In contrast, when dealing with massless partons, the presence of infrared divergences requires a different approach. For this case, reduction methods have been worked out [ 8$]-[14$ which are valid for up to 6 external legs. How to extend these results to more than 6 external legs was not clear until recently [2] due to problems stemming from the inversion of kinematical matrices which were singular if the external momenta were kept in four dimensions. On the other hand, the use of helicity techniques requires 4dimensional external momenta. The main ideas how to overcome this problem will be outlined in the following, leading to reduction formulas for scalar and tensor integrals valid for an arbitrary number of external legs.

\section{Reduction of scalar integrals}

Consider the scalar integral

$$
\begin{aligned}
I_{N}^{n} & =\int \frac{d^{n} k}{i \pi^{n / 2}} \frac{1}{\prod_{l=1}^{N} q_{l}^{2}} \\
q_{l} & =k-r_{l} \quad, \quad r_{l}=\sum_{j=1}^{l} p_{j}
\end{aligned}
$$

in $n=4-2 \epsilon$ dimensions with $N$ massless propagators, depicted in Fig. 1 .

The corresponding expression in Feynman pa- 


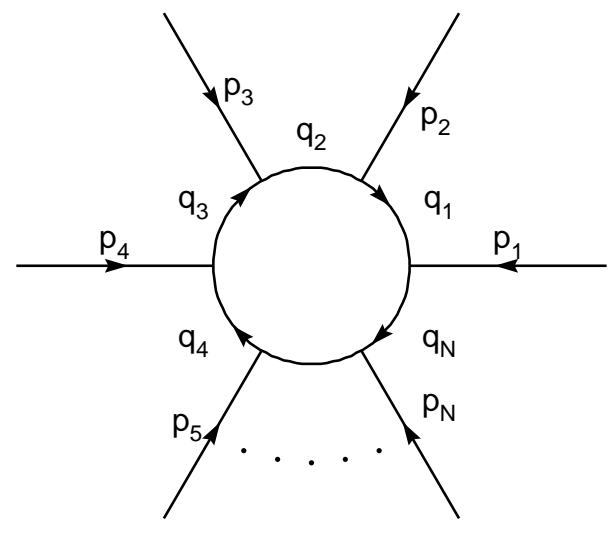

Figure 1. The one-loop N-point graph.

rameter space reads

$$
\begin{aligned}
I_{N}^{n}= & (-1)^{N} \Gamma(N-n / 2) \\
& \times \int_{0}^{\infty} d^{N} z \frac{\delta\left(1-\sum_{l=1}^{N} z_{l}\right)}{(z \cdot S \cdot z)^{N-n / 2}} .
\end{aligned}
$$

The kinematic information is contained in the matrix $S$ which is related to the Gram matrix $G$ by

$$
\begin{aligned}
S_{k l} & =-\frac{1}{2}\left(r_{l}-r_{k}\right)^{2}=\frac{1}{2}\left(G_{k l}-r_{l}^{2}-r_{k}^{2}\right) \\
G_{k l} & =2 r_{k} \cdot r_{l}, \quad k, l=1, \ldots, N .
\end{aligned}
$$

The external momenta are kept in four dimensions; momentum conservation implies $r_{N}=0$. The integral $I_{N}^{n}$ can be split into a singular part with less propagators and a finite, but formally higher dimensional integral as

$$
\begin{aligned}
I_{N}^{n} & =I_{d i v}+I_{f i n} \\
I_{d i v} & =\int \frac{d^{n} k}{i \pi^{n / 2}} \frac{\sum_{l=1}^{N} b_{l} q_{l}^{2}}{\prod_{l=1}^{N} q_{l}^{2}} \\
I_{f i n} & =\int \frac{d^{n} k}{i \pi^{n / 2}} \frac{\left[1-\sum_{l=1}^{N} b_{l} q_{l}^{2}\right]}{\prod_{l=1}^{N} q_{l}^{2}}
\end{aligned}
$$

$$
=-\left(\sum_{l=1}^{N} b_{l}\right)(N-n-1) I_{N}^{n+2},
$$

where the coefficients $b_{l}$ have to fulfill the following equations:

$$
\begin{aligned}
\sum_{l=1}^{N} S_{k l} b_{l} & =-\frac{1}{2} \Leftrightarrow \\
\sum_{l=1}^{N-1} G_{k l} b_{l} & =r_{k}^{2} \sum_{l=1}^{N} b_{l} \quad, \quad \sum_{l=1}^{N-1} r_{l}^{2} b_{l}=1
\end{aligned}
$$

The integral $I_{d i v}$ in (22) is a sum over reduced integrals where one propagator could be cancelled. Our aim is to show that the higher dimensional integrals $I_{N}^{n+2}$ in (3), which are very hard to calculate for $N>4$ and hinder a reduction, drop out for $N>4$. To this end we have to solve Eqs. (4) for the parameters $b_{l}$. Note that for $N=5$, expression (3) is of order $\epsilon$, which means that $I_{\text {fin }}$ can be dropped right away in that case because $(n+2)$-dimensional integrals are finite for $N \geq 4$.

Since only four 4-dimensional external vectors $r_{i}^{\mu}$ can be linearly independent in Minkowski space, $\operatorname{rank}(S)=\min (N, 6)$ and $\operatorname{rank}(G)=\min (N$ $1,4)$. Hence the case $N=6$ is special since $S$ is still invertible for $N=6$, whereas $G$ is not. Therefore Eqs.(1) can still be solved by inverting $S$ for $N=6$, whereas for $N>6$ both, $G$ and $S$ have a vanishing determinant. In this case, the solution to (1) can be constructed in terms of the so-called pseudoinverse $H$ to $G$. It is defined by the properties $H G H=H, G H G=G$. Given a pseudoinverse $H$ a solution to $G x=y$ exists if and only if $y=G H y$. Introducing a basis $E_{l=1, \ldots, 4}^{\mu}$ of Minkowski space and defining the matrices $R$ and $\tilde{G}$ by

$r_{j}^{\mu}=\sum_{l=1}^{4} R_{l j} E_{l}^{\mu}, \quad \tilde{G}_{j k}=2 E_{j} \cdot E_{k}$,

the uniquely defined pseudoinverse is given by

$H=R^{T}\left(R R^{T}\right)^{-1} \tilde{G}^{-1}\left(R R^{T}\right)^{-1} R$.

Now Eq. (4) can be solved for general $N$ [ 2]

${ }^{2}$ In general $H$ is not unique. In our case, the uniqueness follows from the symmetry of $G$. 
Doing so one finds

$$
\sum_{l=1}^{N} b_{l}=0 \quad \text { for } N \geq 6
$$

such that the contribution (3) completely vanishes for all $N \geq 6$. This means that all scalar $N$-point integrals, for arbitrary $N$, can - by recursion - be expressed in terms of box integrals. Explicitly, one finds the following reduction formulas:

For $N \leq 6$, i.e. $\operatorname{det}(S) \neq 0$ :

$$
\begin{aligned}
I_{N}^{n}= & -\frac{1}{2} \sum_{k, l=1}^{N} S_{k l}^{-1} I_{N-1, l}^{n} \\
& +(N-n-1) \frac{\operatorname{det}(G)}{2^{N} \operatorname{det}(S)} I_{N}^{n+2} .
\end{aligned}
$$

For $N>6$, i.e. $\operatorname{det}(S)=0$ :

$$
\begin{aligned}
I_{N}^{n}= & \frac{-1}{v \cdot K \cdot v} \sum_{l=1}^{N-1}\left((K \cdot v)_{l}+\sum_{k=1}^{N-6} \beta_{k} U_{l}^{(k)}\right) \\
& \left(I_{N-1, N}^{n}-I_{N-1, l}^{n}\right) .
\end{aligned}
$$

The reduced integrals $I_{N-1, l}$ are defined as

$$
I_{N-1, l}^{n}=\int \frac{d^{n} k}{i \pi^{n / 2}} \frac{q_{l}^{2}}{\prod_{k=1}^{N} q_{k}^{2}}
$$

and $K=1_{N-1}-H G, \quad v_{l}=r_{l}^{2}$.

The $(N-6) \beta_{k}$ in (8) are free parameters to construct linear combinations of the vectors $\left\{U^{(k)}\right\}(k=1, \ldots, N-6)$ which, together with $U^{(N-5)}$, form a basis for the kernel of $G$. We chose

$$
U^{(N-5)}=K \cdot v /(v \cdot K \cdot v)
$$

parallel to $v$ and the others orthogonal,

$$
v \cdot U^{(k=1, \ldots, N-6)}=0 .
$$

Obviously one can choose the $\beta$ 's such that $(N-$ 6) $b$ 's from the set $\left\{b_{1}, \ldots, b_{N-1}\right\}$ are zero. Doing so one observes that $I_{N}^{n}$ can be expressed by only $6(N-1)$-point graphs for arbitrary $N \geq 6$. This is the generalization to massless kinematics of a similar result in the infrared finite case [6] which has been derived for integer dimensions using 4dimensional Schouten identities.

\section{Reduction of tensor integrals}

Tensor integrals can also be split into a part containing reduced integrals $\left(K_{N}^{\cdots}\right)$ and a part containing higher dimensional objects $\left(J_{N}^{\cdots}\right)$. Using momentum conservation $\left(r_{N}=0\right)$ one can write:

$$
\begin{aligned}
I_{N}^{\mu_{1} \ldots \mu_{L}}= & \int \frac{d^{n} k}{i \pi^{n / 2}} \frac{k^{\mu_{1}} \ldots k^{\mu_{L}}}{\prod_{l=1}^{N} q_{l}^{2}} \\
= & K_{N}^{\mu_{1} \ldots \mu_{L}}+J_{N}^{\mu_{1} \ldots \mu_{L}} \\
K_{N}^{\mu_{1} \ldots \mu_{L}}= & \sum_{l=0}^{L} \sum_{j_{1}, \ldots, j_{l}=1}^{N-1} \mathcal{F}\left(\left\{r_{j}\right\}\right)_{j_{1}, \ldots, j_{l}}^{\mu_{1} \ldots \mu_{L}} \\
& \times I_{N-l, N-j_{1}, \ldots, N-j_{l}}^{n} \mathcal{G}^{N}=\sum_{l=1} \sum_{j_{1}, \ldots, j_{L}-2 l}=1 \\
J_{N}^{\mu_{1} \ldots \mu_{L}} & \times I_{N}^{n+2 l}\left(j_{1}, \ldots, j_{L-2 l}\right)
\end{aligned}
$$

where

$I_{N-l, N-j_{1}, \ldots, N-j_{l}}^{n}=\int \frac{d^{n} k}{i \pi^{n / 2}} \frac{\prod_{m=1}^{l}\left(q_{N}^{2}-q_{j_{m}}^{2}\right)}{\prod_{i=1}^{N} q_{i}^{2}}$

are differences of reduced integrals and

$$
I_{N}^{n+2 l}\left(j_{1}, \ldots, j_{L-2 l}\right)
$$

are higher dimensional integrals with Feynman parameters $z_{j}$ in the numerator. The Lorentz tensors $\mathcal{F}, \mathcal{G}$ present in the above expressions can be written in terms of the objects $\left(v_{l}=r_{l}^{2}\right)$

$$
\begin{aligned}
\mathcal{H}^{\mu \nu} & =r^{\mu} \cdot H \cdot r^{\nu} \\
\mathcal{K}_{l}^{\mu} & =\left(r^{\mu} \cdot H\right)_{l} \\
\mathcal{W}^{\mu} & =\mathcal{K}^{\mu} \cdot v
\end{aligned}
$$

Note that the pseudoinverse $H$ to the Gram matrix $G$ also appears here. The important feature is that one always has

$$
\mathcal{G} \sim g^{\mu \nu} / 2-r^{\mu} \cdot H \cdot r^{\nu} .
$$

If now the Lorentz vectors $r_{j}^{\mu}(j=1, \ldots, N-1)$ span Minkowski space, which is generically the case for $N \geq 5$, one immediately derives, using the explicit expresssion of $H$ given above, that

$$
r^{\mu} \cdot H \cdot r^{\nu}=\left(g^{\mu \nu} / 2\right)^{4-\operatorname{dim}} .
$$


This means

$$
g^{\mu \nu} / 2-r^{\mu} \cdot H \cdot r^{\nu}=\mathcal{O}(\epsilon)
$$

and thus the higher dimensional objects $I_{N}^{n+2 l}$ in Eq. (9) can be dropped for $N \geq 5$. Consequently it follows from Eq. (9) that for the reduction of an $N$-point integral only a small number of higher dimensional integrals is needed, i.e. one has to know $J_{N}^{\mu_{1} \ldots \mu_{L}}$ only for $2 \leq L \leq N=2,3,4$. These can be calculated once and forever, the explicit expressions are

$$
\begin{gathered}
J_{N=2,3,4}^{\mu_{1} \mu_{2}}=-\left(g^{\left.\mu_{1} \mu_{2} / 2-\mathcal{H}^{\mu_{1} \mu_{2}}\right) I_{N}^{n+2}}\right. \\
J_{N=3,4}^{\mu_{1} \mu_{2} \mu_{3}}=-\left[(g / 2-\mathcal{H})^{\cdot \cdot} \mathcal{W}\right]^{\left\{\mu_{1} \mu_{2} \mu_{3}\right\}} I_{N}^{n+2} \\
-\left[(g / 2-\mathcal{H})^{\cdot \cdot} \mathcal{K}_{l}\right]^{\left\{\mu_{1} \mu_{2} \mu_{3}\right\}} I_{N-1, N-l}^{n+2} \\
J_{4}^{\mu_{1} \mu_{2} \mu_{3} \mu_{4}}=\left[(g / 2-\mathcal{H})^{\cdot \cdot}\right. \\
\left.\times(g / 2-\mathcal{H})^{. \cdot}\right]^{\left\{\mu_{1} \mu_{2} \mu_{3} \mu_{4}\right\}} I_{4}^{n+4} \\
-\left[(g / 2-\mathcal{H})^{\cdot \cdot} \mathcal{W} \cdot \mathcal{W} \cdot\right]^{\left\{\mu_{1} \mu_{2} \mu_{3} \mu_{4}\right\}} I_{4}^{n+2} \\
-\frac{1}{2}\left[(g / 2-\mathcal{H})^{\cdot \cdot} \mathcal{W} \cdot \mathcal{K}_{l}\right]^{\left\{\mu_{1} \mu_{2} \mu_{3} \mu_{4}\right\}} I_{3,4-l}^{n+2} \\
-\left[(g / 2-\mathcal{H})^{\cdot \cdot} \mathcal{H} \cdot \mathcal{K}_{l} \cdot\right]^{\left\{\mu_{1} \mu_{2} \mu_{3} \mu_{4}\right\}} I_{3,4-l}^{\nu, n+2} .
\end{gathered}
$$

The indexed bracket is an abbreviation for the sum of all distinguishable distributions of Lorentz indices to the objects inside the bracket, e.g.

$$
\begin{array}{r}
{\left[X Y^{\cdot} Y^{\cdot{ }^{\left\{\mu_{1} \mu_{2} \mu_{3}\right\}}}=X^{\mu_{1}} Y^{\mu_{2}} Y^{\mu_{3}}\right.} \\
+X^{\mu_{2}} Y^{\mu_{3}} Y^{\mu_{1}}+X^{\mu_{3}} Y^{\mu_{1}} Y^{\mu_{2}} .
\end{array}
$$

For the $n$-dimensional part, $K_{N}^{\mu_{1} \ldots \mu_{L}}$, a recursion relation holds. Skipping all the details (see [2]) one finds

$$
\begin{aligned}
K_{N}^{\mu_{1} \ldots \mu_{L}}= & \frac{1}{L}\left[\mathcal{W} \cdot K_{N}^{\{L-1 \mathrm{dots}\}}\right]^{\left\{\mu_{1} \ldots \mu_{L}\right\}} \\
+ & \frac{2^{(L-1)}}{L !}\left[\mathcal{K}_{l} \mathcal{H}_{\nu_{1}} \ldots \mathcal{H}_{\nu_{L-1}}\right]^{\left\{\mu_{1} \ldots \mu_{L}\right\}} \\
& \times I_{N-1, N-l}^{\nu_{1} \ldots \nu_{L-1}} .
\end{aligned}
$$

Putting everything together, the final recursion formula for $N$-point tensor integrals reads

$$
I_{N}^{\mu_{1} \ldots \mu_{L}}=\frac{1}{L}\left[\mathcal{W} \cdot\left(I_{N}-J_{N}\right)^{\{L-1 \operatorname{dots}\}}\right]^{\left\{\mu_{1} \ldots \mu_{L}\right\}}
$$

$$
\begin{aligned}
& +\frac{2^{(L-1)}}{L !}\left[\mathcal{K}_{l} \mathcal{H}_{\nu_{1}} \ldots \mathcal{H}_{\nu_{L-1}}\right]^{\left\{\mu_{1} \ldots \mu_{L}\right\}} \\
& \quad \times I_{N-1, N-l}^{\nu_{1} \ldots \nu_{L-1}}+J_{N}^{\mu_{1} \ldots \mu_{L}} .
\end{aligned}
$$

This form is not immediately suited for iteration, as some reduced integrals do not contain the trivial propagator $k^{2}$ anymore. Therefore one has to shift the loop momenta of the respective integrals by $k \rightarrow k+r_{l}$ before iteration, leading to

$$
\begin{aligned}
& I_{N-1, N-l}^{\mu_{1} \ldots \mu_{P}}(R)= \\
& I_{N-1}^{\mu_{1} \ldots \mu_{P}}\left(\hat{R}_{[N]}\right)-I_{N-1}^{\mu_{1} \ldots \mu_{P}}\left(\hat{R}_{[l]}\right) \\
& =\sum_{k=0}^{P}\left[r_{l}^{\cdot(k)} I_{N-1}^{\{P-k \operatorname{dots}\}}\left(R_{[l]}\right)\right]^{\left\{\mu_{1} \ldots \mu_{P}\right\}} \\
& -I_{N-1}^{\mu_{1} \ldots \mu_{P}}\left(\hat{R}_{[l]}\right) .
\end{aligned}
$$

The argument vectors are

$$
\begin{aligned}
R & =\left(r_{1}, \ldots, r_{N}\right) \\
\hat{R}_{[k]} & =\left(r_{1}, \ldots, \hat{r}_{k}, \ldots, r_{N}\right) \\
R_{[l]} & =\left(r_{l+1}-r_{l}, r_{l+2}-r_{l}, \ldots, r_{N-2+l}-r_{l}, 0\right)
\end{aligned}
$$

where $\hat{r}$ means that the respective vector is missing. The vector indices are understood to be taken cyclically symmetric with periodicity $N$, i.e. $r_{N+l}=r_{l}$ for $l \in\{1, \ldots, N\}$. As we assume $r_{N}=0$, the integrals on the right-hand side of (13) have again at least one trivial propagator and thus are suited for a further reduction step. As a simple example, consider the rank two 3point integral $I_{3}^{\mu_{1} \mu_{2}}(R)$. After the first reduction step and subsequent shift, one obtains

$$
\begin{array}{r}
I_{3}^{\mu_{1} \mu_{2}}(R)=\frac{1}{2}\left(\mathcal{W}^{\mu_{1}} I_{3}^{\mu_{2}}(R)+\mathcal{W}^{\mu_{2}} I_{3}^{\mu_{1}}(R)\right) \\
+\sum_{l=1}^{2}\left(\mathcal{K}_{l}^{\mu_{1}} \mathcal{H}_{\nu}^{\mu_{2}}+\mathcal{K}_{l}^{\mu_{2}} \mathcal{H}_{\nu}^{\mu_{1}}\right) \\
\times\left(r_{l}^{\nu} I_{2}^{n}\left(R_{[l]}\right)+I_{2}^{\nu}\left(R_{[l]}\right)-I_{2}^{\nu}\left(\hat{R}_{[l]}\right)\right) \\
-\left(g^{\mu_{1} \mu_{2}} / 2-\mathcal{H}^{\mu_{1} \mu_{2}}\right) I_{3}^{n+2}(R)
\end{array}
$$

The recursive structure together with the shift can be implemented in an algebraic manipulation program in a straightforward manner. The recursion stops if all tensor integrals are transformed into expressions containing scalar integrals and Lorentz tensors composed of the objects given in 
Eq. (10). Starting with an $N$-point tensor integral one finds a linear combination of scalar integrals with $K \leq N$ legs. To these scalar integrals the reduction formulas (7), (8) can be applied. In this way our reduction formalism solves the problem of calculating massless $N$-point Feynman diagrams for arbitrary $N$. The generalization to the case where some of the propagators are massive will work similarly.

\section{Summary/Outlook}

In conclusion, massless scalar and tensor oneloop integrals for graphs with an arbitrary number of external legs $(N>4)$ can recursively be reduced to linear combinations of (known) box integrals. To deal with the problem of singular Gram matrices we introduced the concept of the pseudoinverse, which renders the whole formalism mathematically well defined for arbitrary $N$. The recursive structure can easily be implemented in algebraic manipulation programs. This allows in principle for the NLO calculation of multi-jet cross sections with a large number of final states. Applications relevant for the near future are e.g. the NLO calculations for $p p \rightarrow b \bar{b} b \bar{b}$ or $p p \rightarrow 4$ jets/photons. The most complicated analytical structure in such a calculation is the scalar 6-point function since, as shown above, no dangerous higher-dimensional objects have to be computed. With the given methods, an explicit analytical expression for the scalar 6-point function has been derived and presented in [2].

\section{Acknowledgments}

We would like to thank the organizers for their work. It was a pleasure for us to take part in the Loops and Legs in Quantum Field Theory 2000 conference. This work was supported by the EU Fourth Training Programme "Training and Mobility of Researchers", Network "Quantum Chromodynamics and the Deep Structure of Elementary Particles", contract FMRX-CT98-0194 (DG 12 - MIHT).

\section{REFERENCES}

1. CDF collaboration, F. Abe et al., Phys. Rev. Lett. 75, 605 (1995).

2. T. Binoth, J.P. Guillet and G. Heinrich, Nucl. Phys. B 572, 361 (2000).

3. D.B. Melrose, Il Nouvo Cimento 40A, 181 (1965).

4. G. Passarino, M. Veltman, Nucl. Phys. B 160, 151 (1979).

5. G.J. van Oldenborgh, J.A.M. Vermaseren, $Z$. Phys. C 46, 425 (1990).

6. W.L. van Neerven, J.A.M. Vermaseren, Phys. Lett. B 137, 241 (1984).

7. A. Denner, Fortsch.Phys. 41, 307 (1993).

8. A.I. Davydychev, Phys. Lett. B 263, 107 (1991).

9. Z. Bern, L. Dixon, D.A. Kosower, Phys. Lett. B 302, 299 (1993); Nucl. Phys. B 412, 751 (1994).

10. J. M. Campbell, E.W.N. Glover, D.J. Miller, Nucl. Phys. B 498, 397 (1997).

11. O.V. Tarasov, Phys. Rev.D 54, 6479 (1996); J. Fleischer, F. Jegerlehner, O.V. Tarasov, Nucl. Phys. B 566, 423 (2000).

12. R.G. Stuart, Comput. Phys. Commun. 48, 367 (1988);

G. Devaraj, R.G. Stuart, Nucl. Phys. B 519, 483 (1998).

13. R. Pittau, Comput. Phys. Commun. 104, 23 (1997); ibid. 111, 48 (1998).

14. S. Weinzierl, Phys. Lett. B 450, 234 (1999). 